TRANSACTIONS OF THE

AMERICAN MATHEMATICAL SOCIETY

Volume 351, Number 2, February 1999, Pages 595-617

S 0002-9947(99)02003-6

\title{
MAXIMAL IDEALS IN MODULAR GROUP ALGEBRAS OF THE FINITARY SYMMETRIC AND ALTERNATING GROUPS
}

\author{
ALEXANDER BARANOV AND ALEXANDER KLESHCHEV
}

\begin{abstract}
The main result of the paper is a description of the maximal ideals in the modular group algebras of the finitary symmetric and alternating groups (provided the characteristic $p$ of the ground field is greater than 2). For the symmetric group there are exactly $p-1$ such ideals and for the alternating group there are $(p-1) / 2$ of them. The description is obtained in terms of the annihilators of certain systems of the 'completely splittable' irreducible modular representations of the finite symmetric and alternating groups. The main tools used in the proofs are the modular branching rules (obtained earlier by the second author) and the 'Mullineux conjecture' proved recently by FordKleshchev and Bessenrodt-Olsson. The results obtained are relevant to the theory of PI-algebras. They are used in a later paper by the authors and A. E. Zalesskii on almost simple group algebras and asymptotic properties of modular representations of symmetric groups.
\end{abstract}

\section{INTRODUCTION}

A permutation of the set $\mathbb{N}=\{1,2,3, \ldots\}$ is called finitary if it fixes all but finitely many elements. The finitary symmetric group $\Sigma_{\infty}$ is the group of all finitary permutations of $\mathbb{N}$. The finitary alternating group $A_{\infty}$ is defined as the group of all even finitary permutations of $\mathbb{N}$. Clearly, $\Sigma_{\infty}$ and $A_{\infty}$ are locally finite groups. They can be represented as the unions

$$
\Sigma_{\infty}=\bigcup_{n \geq 1} \Sigma_{n}, \quad A_{\infty}=\bigcup_{n \geq 1} A_{n}
$$

where $\Sigma_{n}$ and $A_{n}$ are the groups of all permutations and all even permutations of the set $\{1,2, \ldots, n\}$, respectively.

Let $F$ be an arbitrary field of characteristic $p>0$.

The main result of this paper is a description of the maximal two-sided ideals of the group algebras $F \Sigma_{\infty}$ and $F A_{\infty}$ for the case $p>2$. In particular, we show that there are exactly $p-1$ of them in $F \Sigma_{\infty}$ and $\frac{p-1}{2}$ in $F A_{\infty}$.

In the last years there has been a noticeable progress in the theory of group algebras of locally finite groups; see the expository paper [23] and references there. A. E. Zalesskii has shown that the ideals (we always mean two-sided ones) of these algebras are closely related with the so-called inductive systems; see Definition 2.1

Received by the editors November 25, 1996.

1991 Mathematics Subject Classification. Primary 20C05, 16 S34.

Supported by the Fundamental Research Foundation of Belarus and the National Science Foundation. 
below. This idea is crucial in our approach here. It allows one to reduce many problems on ideals of $F \Sigma_{\infty}$ and $F A_{\infty}$ to the ones on modular representations of finite groups $\Sigma_{n}$ and $A_{n}$.

To describe our main results in detail we need some facts and terminology from the modular representation theory of the symmetric group. The main reference here is $[10]$.

Let $\lambda=\left(l_{1} \geq l_{2} \geq \cdots \geq l_{m}>0\right.$ ) be a partition of $n$ (we write $|\lambda|=n$ ). We define

$$
h(\lambda)=m
$$

and

$$
\chi(\lambda)=l_{1}-l_{m}+m .
$$

The irreducible $F \Sigma_{n}$-modules are parametrized by the $p$-regular partitions of $n$. If $\lambda$ is such a partition, we denote by $D^{\lambda}$ the corresponding irreducible. For $s=$ $1,2, \ldots, p-1$ and $n>(p-s)(s-1)$ set

$$
\Phi(s)_{n}=\left\{D^{\mu}|| \mu \mid=n, \quad h(\mu)=s, \quad \chi(\mu) \leq p\right\} .
$$

Let $G$ be a group and $M$ be an $F G$-module. We write $\operatorname{Ann}_{F G}(M)$ for the annihilator of $M$ in $F G$. If $H$ is a subgroup of $G$, we denote by $M \downarrow H$ the restriction of $M$ to $H$.

Theorem 1.1. Let $F$ be a field of characteristic $p>2$.

(i) Set

$$
I(s)_{n}=\bigcap_{D \in \Phi(s)_{n}} \operatorname{Ann}_{F \Sigma_{n}}(D), \quad s=1, \ldots, p-1, \quad n>(p-s)(s-1) .
$$

Then $I(s)_{n}=I(s)_{n+1} \cap F \Sigma_{n}$ for all $s, n$. Moreover,

$$
I(s)=\bigcup_{n>(p-s)(s-1)} I(s)_{n}, \quad s=1, \ldots, p-1,
$$

are exactly all distinct maximal (two-sided) ideals of $F \Sigma_{\infty}$, and $I(s) \cap F \Sigma_{n}=$ (ii) Set $I(s)_{n}$ for $n>(p-s)(s-1)$.

$J(t)_{n}=\bigcap_{D \in \Phi(t)_{n}} \operatorname{Ann}_{F A_{n}}\left(D \downarrow A_{n}\right), \quad t=1, \ldots, \frac{p-1}{2}, \quad n>(p-t)(t-1)$.

Then $J(t)_{n}=J(t)_{n+1} \cap F A_{n}$ for all $t, n$. Moreover,

$$
J(t)=\bigcup_{n>(p-t)(t-1)} J(t)_{n}, \quad t=1, \ldots, \frac{p-1}{2},
$$

are exactly all distinct maximal (two-sided) ideals of $F A_{\infty}$, and $J(t) \cap F A_{n}=$ $J(t)_{n}$ for $n>(p-t)(t-1)$.

(iii) $I(s) \cap F A_{\infty}=J(t)$ where $t=\min (s, p-s), s=1, \ldots, p-1$.

Remark. (1) Theorem 1.1 was conjectured by A. E. Zalesskii.

(2) It is known that every group algebra $F G$ contains the maximal ideal

$$
\operatorname{Aug}(F G)=\left\{\sum_{g \in G} \alpha_{g} g \mid \sum \alpha_{g}=0\right\}
$$


called the augmentation ideal. It has codimension 1 . One can easily demonstrate another maximal ideal of codimension 1 in $F \Sigma_{\infty}$ :

$$
\operatorname{Aug}^{\sigma}\left(F \Sigma_{\infty}\right)=\left\{\sum_{g \in \Sigma_{\infty}} \alpha_{g} g \mid \sum \operatorname{sign}(g) \alpha_{g}=0\right\} .
$$

It can be shown that

$$
\begin{aligned}
I(1) & =\operatorname{Aug}\left(F \Sigma_{\infty}\right), \\
J(1) & =\operatorname{Aug}\left(F A_{\infty}\right), \\
I(p-1) & =\operatorname{Aug}^{\sigma}\left(F \Sigma_{\infty}\right)
\end{aligned}
$$

and that ideals $I(s), 1<s<p-1$, and $J(t), t>1$, are of infinite codimension.

(3) The ideals $I(s)$ of $F \Sigma_{\infty}$ have been essentially described in [15]. Results from [15] are used in this paper.

(4) One can show that all the restrictions $D \downarrow A_{n}$ in the part (ii) of Theorem 1.1 are actually irreducible.

(5) Tensoring $F \Sigma_{n}$-modules with the one-dimensional sign representation is one of the crucial methods used in our proofs. This is why the case $p=2$ happened to be exceptional in this paper. A.E. Zalesskii observed that if $p=2$ there exists at least one maximal ideal in both $F \Sigma_{\infty}$ and $F A_{\infty}$ which is different from the augmentation ideal. But it remains unclear if there are other maximal ideals.

(6) We note that for a ground field of characteristic 0 there is a complete description of the ideal lattice in the group algebra of $\Sigma_{\infty}$ (see $[6,18]$ ). In particular, $\operatorname{Aug}\left(F \Sigma_{\infty}\right)$ and $\operatorname{Aug}^{\sigma}\left(F \Sigma_{\infty}\right)$ are the only maximal ideals of $F \Sigma_{\infty}$. Much less is known about modular group rings. Important information is contained in the papers $[7,19,21,22]$.

(7) The ideals we consider play a role in the theory of identities of algebras (see $[1,18,19,20])$.

(8) In this paper we use recent results from modular representation theory of symmetric groups related with branching rules [13] and tensoring with sign (Mullineux Conjecture) [5], [2].

(9) All results of this paper about the groups $\Sigma_{\infty}$ and $A_{\infty}$ can be easily generalized to the finitary symmetric and alternating groups $\Sigma_{\Omega}$ and $A_{\Omega}$ of an uncountable set $\Omega$. One just has to use inductive limits instead of unions. We leave details to the reader.

\section{ACKNOWLEDGEMENT}

We are grateful to A. E. Zalesskii for stimulating and encouraging discussions.

\section{NOTATION}

F

$\Sigma_{n}\left(\right.$ resp., $\left.A_{n}\right)$

$\mathcal{P}_{n}$

$|\lambda|=n$

$S^{\lambda}$

$D^{\lambda}$ a field of characteristic $p>0$;

the symmetric (resp., alternating) group on $\{1, \ldots, n\}$;

the set of all $p$-regular partitions of $n$;

means " $\lambda$ is a partition of $n$ ";

the Specht module over $F \Sigma_{n}$ corresponding to a partition $\lambda$ of $n[10]$;

the irreducible $F \Sigma_{n}$-module corresponding to a $p$-regular partition $\lambda$ of $n$ [10]; 
$E^{\lambda}, E_{ \pm}^{\mu}$

$\operatorname{sgn}$

$\mathbf{M}: \mathcal{P}_{n} \rightarrow \mathcal{P}_{n}$

$\chi(\lambda), h(\lambda)$

res $A$

$\operatorname{cont}(\lambda)$

denote the irreducible $F A_{n}$-modules (see Section 6 below);

the one-dimensional sign representation of $F G_{n}(g v=\operatorname{sign}(g) v$ for $g \in \Sigma_{n}, v \in \operatorname{sgn}$;

the Mullineux bijection. Thus $D^{\lambda} \otimes \operatorname{sgn} \cong D^{\mathbf{M}(\lambda)}$ (see Section 3 below);

are defined in (1) and (2) above;

the residue of the node $A$, (see [11] and (4) below);

the residue content of the Young diagram $\lambda$ (see [11] and (5) below);

$\lambda_{A}$ stands for the Young diagram $\lambda \backslash\{A\}$ where $\lambda$ is a Young diagram and $A$ is a removable node of $\lambda$ (see Section 3 below).

Let $G$ be a group, $H$ a subgroup of $G, A$ an associative algebra, $M$ an $F G$ module, $S$ a set of $F G$-modules, $N$ an $F H$-module. Then we denote:

$\operatorname{Irr} G$ (resp., Irr $A$ ) the set of the isomorphism classes of the irreducible $F G$ modules (resp., $A$-modules);

Irr $M \quad$ the set of isomorphism classes of the composition factors of $M$;

$\operatorname{Irr} S$ stands for $\bigcup_{M \in S} \operatorname{Irr} M$;

$\operatorname{Irr}(S \downarrow H)$ stands for $\bigcup_{M \in S} \operatorname{Irr}(M \downarrow H)$;

$M \downarrow H\left(\right.$ or $\left.M \downarrow_{H}^{G}\right)$ the restriction of $M$ from $G$ to $H$;

$N \uparrow^{G}\left(\right.$ or $\left.N \uparrow_{H}^{G}\right)$ the module induced from $H$ to $G$.

\section{InduCtive Systems (GENERAL SETting)}

In this section we shortly discuss and push a bit further the relation between inductive systems and (two-sided) ideals in the group algebra of a locally finite group, revealed by A. E. Zalesskii. Assume for simplicity that a locally finite group $G$ is countable. Then $G$ can be represented as a union

$$
G=\bigcup_{i=1}^{\infty} G_{i}
$$

where $G_{1} \subset G_{2} \subset \ldots$ are finite subgroups of $G$ (if $G$ is not countable one has to use an inductive limit).

Definition 2.1. $[23,1.1]$ Let $\Phi_{i}$ be a non-empty subset of $\operatorname{Irr} G_{i}, i=1,2, \ldots$ We say that the collection $\Phi=\left\{\Phi_{i}\right\}_{i \in \mathbb{N}}$ is an inductive system (for $G$ ) if for any $i, j \in \mathbb{N}$ with $i<j$ we have

$$
\Phi_{i}=\operatorname{Irr}\left(\Phi_{j} \downarrow G_{i}\right) .
$$

Let $X$ be a (proper) ideal of the group algebra $F G$. Set

$$
\Phi(X)_{i}=\operatorname{Irr}\left((F G / X) \downarrow G_{i}\right) .
$$

One easily checks that $\Phi(X)=\left\{\Phi(X)_{i}\right\}_{i \in \mathbb{N}}$ is an inductive system. Recall that an ideal $I$ of an algebra $A$ is called primitive if it is the annihilator of an irreducible $A$-module and semiprimitive if it is an intersection of primitive ideals. Equivalently, $I$ is semiprimitive if and only if the Jacobson $\operatorname{radical} \operatorname{Rad}(A / I)$ is trivial. We shall also use the known fact that the Jacobson radical of a locally finite-dimensional algebra is the largest locally nilpotent ideal. The following result is crucial. 
Theorem 2.2 ([23, 8.1, 1.25]).

(i) $\Phi(X)_{i}=\operatorname{Irr}\left(F G_{i} /\left(X \cap F G_{i}\right)\right)$ where $F G_{i} /\left(X \cap F G_{i}\right)$ is considered as an $F G_{i}$-module.

(ii) The map $f: X \mapsto \Phi(X)$ from the set of the proper ideals to the set of inductive systems is surjective.

(iii) Let $\Phi$ be an inductive system. Then the set $f^{-1}(\Phi)$ contains the largest and the smallest (by inclusion) ideals $I(\Phi)$ and $K(\Phi)$, respectively.

(iv) The ideals $I(\Phi)$ are semiprimitive. The quotient algebras $I(\Phi) / K(\Phi)$ are locally nilpotent. In particular, the map $\Phi \rightarrow I(\Phi)$ is a bijection between the set of the inductive systems and the set of the semiprimitive ideals of $F G$.

Definition 2.3. Let $\Phi=\left\{\Phi_{i}\right\}_{i \in \mathbb{N}}$ and $\Psi=\left\{\Psi_{i}\right\}_{i \in \mathbb{N}}$ be inductive systems. We write $\Phi \subseteq \Psi$ if $\Phi_{i} \subseteq \Psi_{i}$ for all $i$.

Lemma 2.4. Let $\Psi_{i} \subseteq \operatorname{Irr} G_{i}, i \in \mathbb{N}$. Assume

$$
\operatorname{Irr}\left(\Psi_{i} \downarrow G_{i-1}\right) \subseteq \Psi_{i-1} .
$$

Set

$$
\tilde{\Psi}_{i}=\bigcap_{j \geq i} \operatorname{Irr}\left(\Psi_{j} \downarrow G_{i}\right) .
$$

Then $\tilde{\Psi}=\left\{\tilde{\Psi}_{i}\right\}_{i \in \mathbb{N}}$ is the largest (with respect to $\subseteq$ ) inductive system contained in $\Psi$.

Proof. Note that

$$
\Psi_{i} \supseteq \operatorname{Irr}\left(\Psi_{i+1} \downarrow G_{i}\right) \supseteq \operatorname{Irr}\left(\Psi_{i+2} \downarrow G_{i}\right) \supseteq \ldots
$$

Since the set $\Psi_{i}$ is finite, there exists $n=n(i)$ such that for any $j>n$ we have

$$
\tilde{\Psi}_{i}=\operatorname{Irr}\left(\Psi_{j} \downarrow G_{i}\right) .
$$

Let $i \in \mathbb{N}$. Choose $j \geq \max (n(i), n(i+1))$. Then

$$
\operatorname{Irr}\left(\tilde{\Psi}_{i+1} \downarrow G_{i}\right)=\operatorname{Irr}\left(\operatorname{Irr}\left(\Psi_{j} \downarrow G_{i+1}\right) \downarrow G_{i}\right)=\operatorname{Irr}\left(\Psi_{j} \downarrow G_{i}\right)=\tilde{\Psi}_{i},
$$

so $\tilde{\Psi}$ is an inductive system. Clearly, it is the largest one contained in $\Psi$.

Definition 2.5. Let $\Phi$ and $\Psi$ be inductive systems. Set $\Theta_{i}=\Phi_{i} \cap \Psi_{i}$. One can easily see that

$$
\operatorname{Irr}\left(\Theta_{i} \downarrow G_{i-1}\right) \subseteq \Theta_{i-1}
$$

In view of Lemma 2.4, there exists the largest inductive system $\tilde{\Theta}$ such that $\tilde{\Theta} \subseteq \Phi$ and $\tilde{\Theta} \subseteq \Psi$. We shall denote $\tilde{\Theta}$ by $\Phi \wedge \Psi$. Moreover, we denote by $\Phi \vee \Psi$ the inductive system with $(\Phi \vee \Psi)_{i}=\Phi_{i} \cup \Psi_{i}$.

\section{Proposition 2.6.}

(i) $\Phi \rightarrow I(\Phi)$ is an order-reversing isomorphism of partially ordered sets between the inductive systems and semiprimitive ideals of $F G$.

(ii) $\Phi \rightarrow I(\Phi)$ is a bijection between the set of the minimal (with respect to $\subseteq$ ) inductive systems and the set of the maximal ideals of $F G$.

(iii) Semiprimitive ideals of FG satisfy A.C.C. if and only if inductive systems satisfy D.C.C.

(iv) For any ideals $I, J$ of $F G$ we have

$$
\Phi(I \cap J)=\Phi(I) \vee \Phi(J) .
$$


(v) For any ideals $I, J$ of $F G$ we have

$$
\Phi(I+J)=\Phi(I) \wedge \Phi(J) .
$$

Proof. We first prove (iv). Fix $k \in \mathbb{N}$ and set

$$
I_{k}=I \cap F G_{k}, \quad J_{k}=J \cap F G_{k} .
$$

In view of Theorem 2.2 (i), we have to show that

$$
\operatorname{Irr}\left(F G_{k} /\left(I_{k} \cap J_{k}\right)\right)=\operatorname{Irr}\left(F G_{k} / I_{k}\right) \cup \operatorname{Irr}\left(F G_{k} / J_{k}\right) .
$$

But this equality follows from the following exact sequences of $G_{k}$-modules:

$$
\begin{aligned}
& 0 \rightarrow I_{k} /\left(I_{k} \cap J_{k}\right) \rightarrow F G_{k} /\left(I_{k} \cap J_{k}\right) \rightarrow F G_{k} / I_{k} \rightarrow 0, \\
& 0 \rightarrow J_{k} /\left(I_{k} \cap J_{k}\right) \rightarrow F G_{k} /\left(I_{k} \cap J_{k}\right) \rightarrow F G_{k} / J_{k} \rightarrow 0,
\end{aligned}
$$

and the embedding $I_{k} /\left(I_{k} \cap J_{k}\right) \cong\left(I_{k}+J_{k}\right) / J_{k} \subset F G_{k} / J_{k}$.

Now we prove (i). By 2.2 (iv), $\Phi \rightarrow I(\Phi)$ is a bijection between the inductive systems and the semiprimitive ideals. It follows from the definitions that $I \supseteq J$ implies $\Phi(I) \subseteq \Phi(J)$. This shows that $I(\Phi) \supseteq I(\Psi)$ implies $\Phi \subseteq \Psi$. Conversely, let $\Phi \subseteq \Psi$. By (iv),

$$
\Phi(I(\Phi) \cap I(\Psi))=\Phi(I(\Phi)) \vee \Phi(I(\Psi))=\Phi \vee \Psi=\Psi,
$$

since $\Phi \subseteq \Psi$. It is known that the intersection of semiprimitive ideals is a semiprimitive ideal. So we have two semiprimitive ideals, $I(\Phi) \cap I(\Psi)$ and $I(\Psi)$, corresponding to one inductive system $\Psi$. In view of 2.2 (iv),

$$
I(\Phi) \cap I(\Psi)=I(\Psi) .
$$

Hence $I(\Phi) \supseteq I(\Psi)$.

(iii) follows immediately from (i).

Let $X$ be a maximal ideal of $F G$. Then $F G / X$ is simple. Observe that $F G / X$ is not locally nilpotent (for example because it contains the identity). Therefore $X$ is semiprimitive. Now (ii) follows from (i) .

(v) For an ideal $X$ of $F G$ we denote by $\bar{X}$ the smallest semiprimitive ideal of $F G$ containing $X$ (it coincides with the intersection of the semiprimitive ideals containing $X)$. Since $I(\Phi(X))$ is semiprimitive and contains $X$, we have $\bar{X} \subseteq$ $I(\Phi(X))$. The inclusions $X \subseteq \bar{X} \subseteq I(\Phi(X))$ imply $\Phi(\bar{X})=\Phi(X)$. By 2.2 (iv), $\bar{X}=I(\Phi(X))$ and $\bar{X} / X$ is locally nilpotent. Set

$$
X=I+J, \quad Y=\bar{I}+\bar{J} .
$$

Observe that

$$
(\bar{I}+X) / X \cong \bar{I} /(\bar{I} \cap X)
$$

is a locally nilpotent ideal in $Y / X$. Analogously $(\bar{J}+X) / X$ is a locally nilpotent ideal in $Y / X$. Since

$$
Y / X=(\bar{I}+X) / X+(\bar{J}+X) / X \text {. }
$$

we conclude that $Y / X$ is locally nilpotent. Hence $\operatorname{Rad}(F G / X) \supseteq Y / X$.

On the other hand, we have $\operatorname{Rad}(F G / X) \subseteq \bar{X} / X$ since $\operatorname{Rad}(F G / \bar{X})=0$. Thus $\bar{X} / X \supseteq Y / X$, whence $\bar{X} \supseteq Y$. Therefore $\bar{X} \supseteq \bar{Y}$. Since $\bar{Y} \supseteq Y \supseteq X$, we conclude that $\bar{X}=\bar{Y}$. So

$$
\Phi(\bar{Y})=\Phi(\bar{X})=\Phi(X) .
$$

Since $\bar{Y}$ is the smallest semiprimitive ideal, containing semiprimitive ideals $\bar{I}$ and $\bar{J}$, (i) implies

$$
\Phi(\bar{Y})=\Phi(\bar{I}) \wedge \Phi(\bar{J}) .
$$


Finally, we get

$$
\Phi(X)=\Phi(\bar{Y})=\Phi(\bar{I}) \wedge \Phi(\bar{J})=\Phi(I) \wedge \Phi(J) .
$$

Remark. In the proof of Proposition 2.6 (v) we have introduced the operation $X \rightarrow$ $\bar{X}$. Note that the set of all (not necessarily proper) semiprimitive ideals of $F G$ is a lattice with respect to the operations $I \cap J$ and $\overline{I+J}$. In view of 2.6 (i) this lattice is antiisomorphic to the lattice of all inductive systems with respect to the operations $\vee$ and $\wedge$, if we admit the empty inductive system (which will correspond to the ideal $F G$ ).

Definition 2.7. An inductive system $\Phi=\left\{\Phi_{i}\right\}_{i \in \mathbb{N}}$ is called semisimple if for any $i \in \mathbb{N}$ and any $D \in \Phi_{i+1}$ the restriction $D \downarrow G_{i}$ is completely reducible.

Lemma 2.8. Let $\Phi=\left\{\Phi_{i}\right\}_{i \in \mathbb{N}}$ be an inductive system for $G$. Then $\Phi$ is semisimple if and only if

$$
I(\Phi) \cap F G_{i}=\bigcap_{D \in \Phi_{i}} \operatorname{Ann}_{F G_{i}} D
$$

for all $i \in \mathbb{N}$.

Proof. Set

$$
X_{i}=\bigcap_{D \in \Phi_{i}} \operatorname{Ann}_{F G_{i}} D, \quad i=1,2, \ldots
$$

Let $\Phi$ be semisimple. If $i<j$ and $D \in \Phi_{j}$, we have $D \downarrow G_{i}=D_{1} \oplus \cdots \oplus D_{s}$ with all $D_{k} \in \Phi_{i}$. Moreover, $\operatorname{Irr}\left(\Phi_{j} \downarrow G_{i}\right)=\Phi_{i}$, and the annihilator of a direct sum of modules is the intersection of their annihilators. So we have

$$
\begin{aligned}
X_{j} \cap F G_{i} & =\left(\bigcap_{D \in \Phi_{j}} \operatorname{Ann}_{F G_{j}} D\right) \cap F G_{i}=\bigcap_{D \in \Phi_{j}}\left(\left(\operatorname{Ann}_{F G_{j}} D\right) \cap F G_{i}\right) \\
& =\bigcap_{D \in \Phi_{j}} \operatorname{Ann}_{F G_{i}}\left(D \downarrow G_{i}\right)=X_{i} .
\end{aligned}
$$

Therefore $X_{i} \subseteq X_{j}$ (we consider both $X_{i}$ and $X_{j}$ as subsets of $F G$ via embeddings $\left.F G_{l} \subset F G\right)$. So $X=\bigcup_{i=1}^{\infty} X_{i}$ is an ideal of $F G$ such that $X \cap F G_{i}=X_{i}$. So, taking into account Theorem 2.2 (i), we get

$$
\Phi(X)_{i}=\operatorname{Irr}\left(F G_{i} /\left(F G_{i} \cap X\right)\right)=\operatorname{Irr}\left(F G_{i} / X_{i}\right)=\operatorname{Irr}\left(F G_{i} / \operatorname{Ann}_{F G_{i}}\left(\bigoplus_{D \in \Phi_{i}} D\right)\right)=\Phi_{i}
$$

i.e. $\Phi(X)=\Phi$.

Now note that

$$
F G / X=\bigcup_{i=1}^{\infty} F G_{i} /\left(F G_{i} \cap X\right)=\bigcup_{i=1}^{\infty} F G_{i} / X_{i} .
$$

Since every ideal $X_{i}$ is an intersection of primitive ideals (annihilators of irreducible modules) it is semiprimitive, i.e. $F G_{i} / X_{i}$ is semisimple. It follows that $\operatorname{Rad}(F G / X)=0$, i.e. $X$ is semiprimitive. By Theorem 2.2 (iv), $X=I(\Phi)$, which proves the equality (3).

Conversely, let $I(\Phi) \cap F G_{i}=X_{i}, i=1,2, \ldots$ For any $i \in \mathbb{N}$, we consider an arbitrary module $D \in \Phi_{i+1}$. Note that $X_{i} \subseteq X_{i+1} \subseteq \operatorname{Ann}_{F G_{i+1}}(D)$ by assumption. 
Hence the restriction $D \downarrow G_{i}$ is an $F G_{i} / X_{i}$-module. However, $F G_{i} / X_{i}$ is a semisimple finite-dimensional algebra (because $X_{i} \supseteq \bigcap_{D \in \operatorname{Irr} G_{i}} \operatorname{Ann}_{F G_{i}} D=\operatorname{Rad} F G_{i}$ ). Therefore $D \downarrow G_{i}$ is semisimple.

\section{Modular Representations of Symmetric Groups}

We start from some notation concerning Young diagrams.

Let us fix an arbitrary partition $\lambda=\left(l_{1} \geq \cdots \geq l_{m}>0\right)$ of $n$. We do not distinguish between $\lambda$ and its Young diagram which is defined as a subset

$$
\left\{(i, j) \mid 1 \leq i \leq m, \quad 1 \leq j \leq l_{i}\right\}
$$

of $\mathbb{N} \times \mathbb{N}$. Elements $(i, j)$ of $\mathbb{N} \times \mathbb{N}$ are called nodes. If $A=(i, j)$ is a node, we define

$$
\text { res } A=(j-i) \quad(\bmod p) .
$$

If a diagram $\lambda$ contains exactly $c_{i}$ nodes of residue $i, i=0,1, \ldots, p-1$, we define

$$
\operatorname{cont}(\lambda)=\left(c_{0}, c_{1}, \ldots, c_{p-1}\right) .
$$

If $l_{i}>l_{i+1}$, then the node $\left(i, l_{i}\right)$ is called a removable node for $\lambda\left(l_{m+1}\right.$ is interpreted as 0$)$. If $A=\left(i, l_{i}\right)$ is a removable node for $\lambda$, we denote by $\lambda_{A}$ the partition $\left(l_{1}, \ldots, l_{i-1}, l_{i}-1, l_{i+1}, \ldots, l_{m}\right)$ of $n-1$ whose Young diagram is $\lambda \backslash\{A\}$. We call $(i, j)$ an indent node for $\lambda$ if $l_{i}<l_{i-1}, j=l_{i}+1$ ( $l_{0}$ is interpreted as $\left.+\infty\right)$.

We list some known results on representations of $\Sigma_{n}$ for future reference. All modules over $\Sigma_{n}$ are $F \Sigma_{n}$-modules.

The Specht module $S^{\lambda}$ labelled by partitions $\lambda$ of $n$ are defined in [10, Section 4]. The irreducible modules $D^{\lambda}$ labelled by the $p$-regular partitions $\lambda$ of $n$ are defined as the top composition factors of the corresponding $S^{\lambda}$ (see [10, Section 11]). So

Lemma 3.1. Let $\lambda \in \mathcal{P}_{n}$. Then $D^{\lambda} \in \operatorname{Irr}\left(S^{\lambda}\right)$.

The following well known result follows from the "Nakayama Conjecture" [11, 6.1] and Lemma 3.1.

Lemma 3.2. If $D^{\mu} \in \operatorname{Irr}\left(S^{\lambda}\right)$, then

$$
\operatorname{cont}(\mu)=\operatorname{cont}(\lambda)
$$

We need the following Branching Theorem for the Specht modules.

Theorem 3.3. $[10,9.3]$ The restriction $S^{\lambda} \downarrow \Sigma_{n-1}$ has a filtration whose factors are

$$
\left\{S^{\lambda_{A}} \mid A \text { is a removable node for } \lambda\right\} \text {. }
$$

We shall use a special case of the branching rule for irreducible modules obtained in [13]. To state the result we need a concept of a normal node.

Definition 3.4. Let $A=\left(i, l_{i}\right)$ be a removable node for $\lambda$. We call $A$ normal (for $\lambda$ ) if and only if for every $j=1,2, \ldots, i-1$ the following condition holds: the number of the removable nodes $B$ in the rows $j, j+1, \ldots, i-1$ such that res $B=\operatorname{res} A$ is greater than or equal to the number of the indent nodes $C$ in the rows $j, j+1, \ldots, i-1$ such that $\operatorname{res} C=\operatorname{res} A$.

Theorem 3.5. $[13,16]$ Let $\lambda \in \mathcal{P}_{n}$ and $A$ be a removable node of $\lambda$ such that $\lambda_{A} \in \mathcal{P}_{n-1}$. Then $D^{\lambda_{A}} \in \operatorname{Irr}\left(D^{\lambda} \downarrow \Sigma_{n-1}\right)$ if and only if $A$ is normal. $d$.

If $d \in \mathbb{N}$, we define the $p$-exponent of $d$ as the largest $i \in \mathbb{Z}_{+}$such that $p^{i}$ divides 
Theorem 3.6. $[9,12]$ Let $\lambda \in \mathcal{P}_{n}$. Then $S^{\lambda}$ is irreducible if and only if the $p$ exponents of the hook length are constant along the columns of $\lambda$.

We denote by sgn the one-dimensional sign representation of $\Sigma_{n}$. The Mullineux bijection $\mathbf{M}: \mathcal{P}_{n} \rightarrow \mathcal{P}_{n}$ is defined via

$$
D^{\lambda} \otimes \operatorname{sgn} \cong D^{\mathbf{M}(\lambda)} .
$$

The main result of [5] and [2] provides an algorithm for calculating $\mathbf{M}(\lambda)$ which we now describe.

The rim of a Young diagram $\lambda$ is its south-east border - in other words, a node $(i, j)$ of $\lambda$ belongs to the rim if and only if $(i+1, j+1) \notin \lambda$.

Let us number the nodes of the rim moving from the "top-right" to the "leftbottom" (see the example below). Define the first $p$-segment of the rim as the set consisting of the nodes with numbers $\leq p$. If the last node $B$ of the first $p$-segment is in the last row of $\lambda$, then $\lambda$ has only one $p$-segment. If not, let $r$ be the row containing $B$. The first node of the second $p$-segment is the node which has the smallest number, say $d$, among the nodes of the rim in row $r+1$. The second $p$-segment is now defined as the set consisting of the nodes whose numbers $i$ satisfy $d \leq i \leq d+p-1$. Repeating this procedure sufficiently many times we reach the bottom row of the diagram. It is clear that all $p$-segments except possibly the last one contain $p$ nodes. The $p$-edge is defined as the union of the $p$-segments.

Now define diagrams $\lambda^{(1)}, \lambda^{(2)}, \lambda^{(3)}, \ldots$ as follows.

$$
\lambda^{(1)}=\lambda, \quad \lambda^{(i)}=\lambda^{(i-1)} \backslash\left\{p-\text { edge of } \lambda^{(i-1)}\right\}
$$

for $i>1$. We choose $z$ to be maximal with respect to $\lambda^{(z)} \neq \emptyset$. The Mullineux symbol of $\lambda$ is an array

$$
G(\lambda)=\left(\begin{array}{cccc}
a_{1} & a_{2} & \ldots & a_{z} \\
r_{1} & r_{2} & \ldots & r_{z}
\end{array}\right)
$$

where $a_{i}$ is the number of nodes of the $p$-edge of $\lambda^{(i)}$, and $r_{i}=h\left(\lambda^{(i)}\right)$ is the number of rows in $\lambda^{(i)}, i=1,2, \ldots, z$.

The following theorem was first proved in [5]. An easier proof was recently found by Bessenrodt and Olsson [2].

Theorem 3.7. Let $\lambda \in \mathcal{P}_{n}$, and

$$
G(\lambda)=\left(\begin{array}{llll}
a_{1} & a_{2} & \ldots & a_{z} \\
r_{1} & r_{2} & \ldots & r_{z}
\end{array}\right)
$$

Put $\varepsilon_{i}=0$ if $p \mid a_{i}$, and $\varepsilon_{i}=1$ otherwise. Then

$$
G(\mathbf{M}(\lambda))=\left(\begin{array}{llll}
a_{1} & a_{2} & \ldots & a_{z} \\
s_{1} & s_{2} & \ldots & s_{z}
\end{array}\right)
$$

where $s_{i}=a_{i}+\varepsilon_{i}-r_{i}, i=1,2, \ldots, z$.

Thus the Mullineux symbol of $\mathbf{M}(\lambda)$ can be easily found from that of $\lambda$. On the other hand, a $p$-regular partition can be reconstructed from its Mullineux symbolwe just add $p$-edges starting from the last one. 
Example. Let $\lambda=\left(6,4^{2}, 2,1\right)$. Then the rim of $\lambda$ contains the nodes represented by numbers in the following picture.

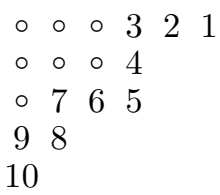

Let $p=5$. The nodes of the $p$-edge (which consists of two $p$-segments) are coloured in black in the following picture.

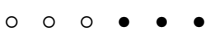

$\circ \quad 0 \quad 0$

$\circ \circ \circ$

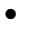

We have $\lambda^{(2)}=\left(3^{3}\right), \lambda^{(3)}=\left(2^{2}\right), \lambda^{(4)}=(1), z=4$, and

$$
G(\lambda)=\left(\begin{array}{llll}
8 & 5 & 3 & 1 \\
5 & 3 & 2 & 1
\end{array}\right), \quad G(\mathbf{M}(\lambda))=\left(\begin{array}{llll}
8 & 5 & 3 & 1 \\
4 & 2 & 2 & 1
\end{array}\right) .
$$

Finally, $\mathbf{M}(\lambda)=\left(7^{2}, 2,1\right)$.

\section{Some lemmas on BRAnChing AND tensoring With Sign}

Let $m, f \in \mathbb{N}$. Represent $m$ in the form

$$
m=(p-1) d+r, \quad d \in \mathbb{Z}, \quad 0<r \leq p-1 .
$$

We need to consider certain classes of partitions. Set

$$
\begin{aligned}
\beta(m, f) & =\left((f+d)^{p-1},(f+d-1)^{p-1}, \ldots,(f+1)^{p-1}, f^{r}\right) ; \\
\alpha(m, f) & =\left((f+d)^{r},(f+d-1)^{p-1},(f+d-2)^{p-1}, \ldots, f^{p-1}\right) ; \\
\alpha(m) & =\alpha(m, 1) .
\end{aligned}
$$

Obviously $h(\beta(m, f))=h(\alpha(m, f))=m$.

$$
\begin{aligned}
& \text { If } \mu=\left(\mu_{1}, \ldots, \mu_{t}\right) \text { is a } p \text {-regular partition and } f>\mu_{1} \text {, put } \\
& \begin{array}{l}
\beta(m, f ; \mu)=\left((f+d)^{p-1},(f+d-1)^{p-1}, \ldots,(f+1)^{p-1}, f^{r}, \mu_{1}, \ldots, \mu_{t}\right) ; \\
\alpha(m, f ; \mu)=\left((f+d)^{r},(f+d-1)^{p-1},(f+d-2)^{p-1}, \ldots, f^{p-1}, \mu_{1}, \ldots, \mu_{t}\right)
\end{array}
\end{aligned}
$$

(glue $\mu$ to the bottom of $\beta(m, f)$ and $\alpha(m, f)$, respectively).

Lemma 4.1. The Specht module $S^{\alpha(m)}$ is irreducible.

Proof. This follows from Theorem 3.6.

Let us fix a $p$-regular partition $\lambda$ with $k$ distinct parts, i.e.

$$
\lambda=\left(l_{1}^{a_{1}}, l_{2}^{a_{2}}, \ldots, l_{k}^{a_{k}}\right), \quad l_{1}>l_{2}>\cdots>l_{k}>0, \quad 0<a_{i}<p .
$$

Then $\lambda$ has $k$ removable and $k+1$ indent nodes. Let $A_{1}, \ldots, A_{k}$ (resp., $B_{1}, \ldots, B_{k+1}$ ) be all removable (resp., indent) nodes of $\lambda$ numbered from top to bottom, i.e. $A_{i}=\left(a_{1}+\cdots+a_{i}, l_{i}\right), B_{j}=\left(a_{1}+\cdots+a_{j-1}+1, l_{j}+1\right), i=1, \ldots, k, j=1, \ldots, k+1$ $\left(l_{k+1}\right.$ is interpreted as 0$)$.

Lemma 4.2. Assume $\operatorname{res} A_{1}=\operatorname{res} A_{2}=\cdots=\operatorname{res} A_{r}$ for some $r \in\{1, \ldots, k\}$. Then $A_{1}, A_{2}, \ldots, A_{r}$ are normal nodes. 
Proof. Since $\lambda$ is $p$-regular, we have res $A_{i} \neq \operatorname{res} B_{i}$ for all $i=1, \ldots, k$. Now use 3.4 .

Lemma 4.3. Let $r \geq 1$ be the largest integer such that

$$
l_{1}=l_{2}+1=l_{3}+2=\cdots=l_{r}+(r-1)
$$

and

$$
a_{2}=a_{3}=\cdots=a_{r}=(p-1) .
$$

Then $r$ is minimal such that $\lambda_{A_{r}}$ is p-regular. Moreover, $A_{r}$ is normal for $\lambda$.

Proof. The first part of the lemma follows from the definitions. To prove that $A_{r}$ is normal it suffices to note that

$$
\operatorname{res} A_{1}=\operatorname{res} A_{2}=\cdots=\operatorname{res} A_{r}
$$

and use Lemma 4.2.

Corollary 4.4. Let $\lambda=\left(l_{1}^{a_{1}}, l_{2}^{a_{2}}, \ldots, l_{k}^{a_{k}}\right) \in \mathcal{P}_{n}$, with $l_{k}=1$. Assume that the bottom removable node $A_{k}$ is the only removable node $A_{i}$ such that $\lambda_{A_{i}}$ is p-regular. Then $\lambda=\alpha\left(a_{1}+a_{2}+\cdots+a_{k}\right)$.

Proof. It follows from Lemma 4.3 that

$$
l_{i}=l_{k}+(k-i), \quad i=1,2, \ldots, k,
$$

and

as desired.

$$
a_{2}=a_{3}=\cdots=a_{k}=p-1,
$$

Definition 4.5. Let $\lambda$ be a $p$-regular partition. We denote by $A(\lambda)$ the top removable node of $\lambda$ such that $\lambda_{A(\lambda)}$ is $p$-regular. Define

$$
\varphi(\lambda)=\lambda_{A(\lambda)} .
$$

Lemma 4.6. Let $\lambda \in \mathcal{P}_{n}$. Then

$$
D^{\varphi(\lambda)} \in \operatorname{Irr}\left(D^{\lambda} \downarrow \Sigma_{n-1}\right) .
$$

Proof. By Lemma 4.3, $A(\lambda)$ is normal for $\lambda$. So it suffices to apply Theorem 3.5.

Remark. Lemma 4.6 is known [8]. We reprove it here for completeness only. Note however that the result is insufficient to prove Proposition 4.14. There we do have to use Theorem 3.5.

Lemma 4.7. Let $\mu$ be a p-regular partition. Assume that for some $m$ we have $\alpha(m) \subseteq \mu$. Set $n=|\alpha(m)|$. Then

$$
D^{\alpha(m)} \in \operatorname{Irr}\left(D^{\mu} \downarrow \Sigma_{n}\right)
$$

Proof. In view of Lemma 4.6, it suffices to show that $\alpha(m)=\varphi^{i}(\mu)$ for some $i$. Since $\varphi^{|\mu|}(\mu)=\emptyset$ and $(m, 1) \in \alpha(m) \subseteq \mu$, there exists $i$ such that $A\left(\varphi^{i}(\mu)\right)=(m, 1)$. So $(m, 1)$ is the top removable node of $\varphi^{i}(\mu)$ leaving a $p$-regular diagram after its removal. Moreover, $(m, 1)$ belongs to the first column, so it is the bottom removable node of $\varphi^{i}(\mu)$. By Corollary 4.4 we now get $\varphi^{i}(\mu)=\alpha(m)$.

Proposition 4.8. Let $n \in \mathbb{N}$ and $\lambda$ be a p-regular partition with $h(\lambda) \geq n(p-1)$. Then

$$
\operatorname{Irr}\left(D^{\lambda} \downarrow \Sigma_{n}\right)=\operatorname{Irr} \Sigma_{n}
$$


Proof. Since $\lambda$ is $p$-regular and $h(\lambda) \geq n(p-1)$, we have $\alpha(n(p-1)) \subseteq \lambda$. Let $j=|\alpha(n(p-1))|$. Then Lemma 4.7 implies

$$
D^{\alpha(n(p-1))} \in \operatorname{Irr}\left(D^{\lambda} \downarrow \Sigma_{j}\right) .
$$

Let $\mu \in \mathcal{P}_{n}$. Then $\mu \subseteq \alpha(n(p-1))$. In view of Lemma 4.1,

$$
D^{\alpha(n(p-1))}=S^{\alpha(n(p-1))} .
$$

Now Theorem 3.3 implies that $D^{\alpha(n(p-1))} \downarrow \Sigma_{n}$ has a filtration such that $S^{\mu}$ occurs as its factor. Since $\mu$ is $p$-regular, $D^{\mu} \in \operatorname{Irr}\left(S^{\mu}\right)$ by Lemma 3.1, and the result follows.

We need to somewhat develop Lemma 4.7. Our goal now is Proposition 4.14.

Lemma 4.9. Let $\mu=\left(\mu_{1}, \ldots, \mu_{s}\right)$ be a p-regular partition ( $\mu$ may be $\emptyset$ ) and $f$ be an integer with $f>\mu_{1}+1$. Set $\alpha=\alpha(m, f ; \mu), \beta=\beta(m, f-1 ; \mu), n=|\beta|$. Then

$$
D^{\beta} \in \operatorname{Irr}\left(D^{\alpha} \downarrow \Sigma_{n}\right) .
$$

Proof. Let $m=(p-1) d+r, d \in \mathbb{Z}, 0<r \leq p-1$. Define the partitions $\alpha^{(0)}, \alpha^{(1)}, \ldots, \alpha^{(r)}$ and the sets $M_{0}, M_{1}, \ldots, M_{r-1}$ of nodes inductively as follows. Set $\alpha^{(0)}=\alpha$ and $\alpha^{(i+1)}=\alpha^{(i)} \backslash M_{i}$ where $M_{i}$ is the set of the top $(d+1)$ removable nodes of $\alpha^{(i)}, i=0,1, \ldots, r-1$. Note that all nodes of the set $M_{i}$ are of the same residue. So Lemma 4.2 implies that the nodes of $M_{i}$ are normal nodes for the partition $\alpha^{(i)}(i=0,1, \ldots, r-1)$. Therefore

$$
D^{\alpha^{(i+1)}} \in \operatorname{Irr}\left(D^{\alpha^{(i)}} \downarrow \Sigma_{\left|\alpha^{(i+1)}\right|}\right)
$$

for all $i=0,1, \ldots, r-1$. It remains to observe that $\alpha^{(r)}=\beta$.

Lemma 4.10. Let $\mu=\left(\mu_{1}, \ldots, \mu_{s}\right)$ be a p-regular partition ( $\mu$ may be $\left.\emptyset\right), f>$ $\mu_{1}+1$. Set $\beta=\beta(m, f ; \mu), \gamma=\beta(m, f-1 ; \mu), n=|\gamma|$. Then

$$
D^{\gamma} \in \operatorname{Irr}\left(D^{\beta} \downarrow \Sigma_{n}\right) \text {. }
$$

Proof. The proof is similar to that of Lemma 4.9.

Lemma 4.11. Let $\mu=\left(\mu_{1}, \ldots, \mu_{s}\right)$ be a p-regular partition, $f>\mu_{1}+1$. Set $\nu=\varphi(\mu)$ (see 4.5), $\beta=\beta(m, f ; \mu), \gamma=\beta(m, f-1 ; \nu), n=|\gamma|$. Then

$$
D^{\gamma} \in \operatorname{Irr}\left(D^{\beta} \downarrow \Sigma_{n}\right) \text {. }
$$

Proof. Let $m=d(p-1)+r, d \in \mathbb{Z}, 0<r \leq p-1$. It follows from the definition of $\beta$ that in the first $m$ rows there are exactly $d+1$ indent nodes for $\beta$. Moreover, these indent nodes all have the same residue, say $\omega$. Let $A$ be the removable node for $\beta$ defined from the equality

$$
\beta=\beta(m, f ; \nu) \sqcup\{A\} .
$$

Assume res $A \neq \omega$. Then $A$ is normal for $\beta$. Therefore, setting $\beta^{\prime}=\beta(m, f ; \nu)$, $n^{\prime}=\left|\beta^{\prime}\right|$, we have

$$
D^{\beta^{\prime}} \in \operatorname{Irr}\left(D^{\beta} \downarrow \Sigma_{n^{\prime}}\right),
$$

in view of Theorem 3.5. Moreover, Lemma 4.10 implies $D^{\gamma} \in \operatorname{Irr}\left(D^{\beta^{\prime}} \downarrow \Sigma_{n}\right)$, and we are done.

Now let res $A=\omega$. Put $\beta^{\prime \prime}=\beta(m, f-1, \mu), n^{\prime \prime}=\left|\beta^{\prime \prime}\right|$. (Actually $n^{\prime \prime}=n+1$.) By Lemma $4.10, D^{\beta^{\prime \prime}} \in \operatorname{Irr}\left(D^{\beta} \downarrow \Sigma_{n^{\prime \prime}}\right)$. Moreover, the removable nodes for $\beta^{\prime \prime}$ in the 
first $m$ rows all have residue $\omega-1$. So $A$ is normal for $\beta^{\prime \prime}$. Since $\gamma=\beta_{A}^{\prime \prime}$, we get from Theorem 3.5

$$
D^{\gamma} \in \operatorname{Irr}\left(D^{\beta^{\prime \prime}} \downarrow \Sigma_{n}\right)
$$

and the result follows.

Lemma 4.12. Let $\mu$ be a p-regular partition and $f$ be an integer with $f>|\mu|+1$. Set $\beta=\beta(m, f ; \mu), e=f-|\mu|, \gamma=\beta(m, e), n=|\gamma|$. Then

$$
D^{\gamma} \in \operatorname{Irr}\left(D^{\beta} \downarrow \Sigma_{n}\right) .
$$

Proof. Apply Lemma 4.11 sufficiently many times.

The following result is a generalization of Lemma 4.7.

Lemma 4.13. Let

$$
\lambda=\left(l_{1}, l_{2}, \ldots, l_{m}, l_{m+1}, \ldots, l_{s}\right)
$$

be a p-regular partition, with $l_{m}>l_{m+1}$. Set

$$
\begin{aligned}
\mu & =\left(l_{m+1}, \ldots, l_{s}\right), \\
\alpha & =\alpha\left(m, l_{m} ; \mu\right), \quad n=|\alpha|
\end{aligned}
$$

$(\mu=\emptyset$ if $m=s)$. Then

$$
D^{\alpha} \in \operatorname{Irr}\left(D^{\lambda} \downarrow \Sigma_{n}\right)
$$

Proof. We choose the maximal $i \geq 0$ such that $\varphi^{i}(\lambda)$ has the form

$$
\varphi^{i}(\lambda)=\left(k_{1}, \ldots, k_{m-1}, l_{m}, \ldots, l_{s}\right),
$$

see Definition 4.5. This means that the node $A\left(\varphi^{i}(\lambda)\right)$ lies in the $m$-th row or below. Since $l_{m} \neq l_{m+1}$, it follows from Lemma 4.3 that $\left(k_{1}, \ldots, k_{m-1}, l_{m}\right)=\alpha\left(m, l_{m}\right)$. Therefore $\varphi^{i}(\lambda)=\alpha$, and the result follows from Lemma 4.6.

Proposition 4.14. Let $\lambda=\left(l_{1}, l_{2}, \ldots, l_{s}\right)$ be a p-regular partition, $m \in\{1,2, \ldots$, $s\}$, and

$$
l_{m}>2+\sum_{i=m+1}^{s} l_{i}
$$

Set $f=l_{m}-1-\sum_{i=m+1}^{s} l_{i}, \beta=\beta(m, f), n=|\beta|$. Then

$$
D^{\beta} \in \operatorname{Irr}\left(D^{\lambda} \downarrow \Sigma_{n}\right) \text {. }
$$

Proof. Set

$$
\begin{aligned}
\mu & =\left(l_{m+1}, \ldots, l_{s}\right), \\
\alpha & =\alpha\left(m, l_{m} ; \mu\right), \\
n_{1} & =|\alpha| .
\end{aligned}
$$

By Lemma 4.13,

$$
D^{\alpha} \in \operatorname{Irr}\left(D^{\lambda} \downarrow \Sigma_{n_{1}}\right)
$$

Let

$$
\beta_{1}=\beta\left(m, l_{m}-1 ; \mu\right), \quad n_{2}=\left|\beta_{1}\right|
$$

Then

$$
D^{\beta_{1}} \in \operatorname{Irr}\left(D^{\alpha} \downarrow \Sigma_{n_{2}}\right)
$$

by Lemma 4.9. Now apply Lemma 4.12 with $\beta=\beta_{1}$ and $\gamma=\beta$. 
Lemma 4.15. Let $\lambda \in \mathcal{P}_{n}$ and

$$
\operatorname{cont}(\lambda)=\left(c_{0}, c_{1}, \ldots, c_{p-1}\right) .
$$

Assume that $D^{\mu} \in \operatorname{Irr}\left(D^{\lambda} \downarrow \Sigma_{n-1}\right)$ and

$$
\operatorname{cont}(\mu)=\left(d_{0}, d_{1}, \ldots, d_{p-1}\right) .
$$

Then there exists $j \in\{0,1, \ldots, p-1\}$ such that $d_{j}=c_{j}-1$ and $d_{i}=c_{i}$ for $i \neq j$.

Proof. By Theorem 3.3, the restriction $S^{\lambda} \downarrow \Sigma_{n-1}$ has a filtration with factors $S^{\lambda_{A}}$ where $A$ is a removable node for $\lambda$. Note that

$$
\operatorname{cont}\left(\lambda_{A}\right)=\left(c_{0}, \ldots, c_{j}-1, \ldots, c_{p-1}\right)
$$

if res $A=j$. By $3.1, D^{\lambda} \in \operatorname{Irr}\left(S^{\lambda}\right)$ so

$$
\operatorname{Irr}\left(D^{\lambda} \downarrow \Sigma_{n-1}\right) \subseteq \operatorname{Irr}\left(S^{\lambda} \downarrow \Sigma_{n-1}\right)=\bigcup_{A} \operatorname{Irr}\left(S^{\lambda_{A}}\right) .
$$

Now it remains to use Lemma 3.2.

Remark. Actually, much more is known about $\operatorname{Irr}\left(D^{\lambda} \downarrow \Sigma_{n-1}\right)$. For example, the contents of the factors $\operatorname{Irr}\left(D^{\lambda} \downarrow \Sigma_{n-1}\right)$ are described in [14]. See also [16] for more information. One can use the argument from the proof of 4.15 to show that $D^{\mu} \in$ $\operatorname{Irr}\left(D^{\lambda} \downarrow \Sigma_{n-1}\right)$ implies $\mu$ dominates some $\lambda_{A}$.

The rest of this section is devoted to certain results concerning the Mullineux map described at the end of Section 3.

Lemma 4.16. Let $e, m \in \mathbb{N}, \beta=\beta(m, p e)$. Represent $m$ in the form

$$
m=(p-1) d+r, \quad d \in \mathbb{Z}, \quad 0<r \leq p-1 .
$$

Let $\mathbf{M}(\beta)=\gamma=\left(\gamma_{1} \geq \gamma_{2} \geq \cdots \geq \gamma_{s}>0\right)$. Then $s=p+d-r$ and $\gamma_{s} \geq r e$.

Proof. One can easily see that the Mullineux symbol of $\beta$ has the form

$$
G(\beta)=\left(\begin{array}{cccc}
(d+1) p & (d+1) p & (d+1) p & \\
\underbrace{d(p-1)+r}_{r e \text { times }} & d(p-1)+r & \cdots & d(p-1)+r
\end{array} \text { the rest }\right) .
$$

So, by Theorem 3.7, the Mullineux symbol $G(\gamma)$ has the form

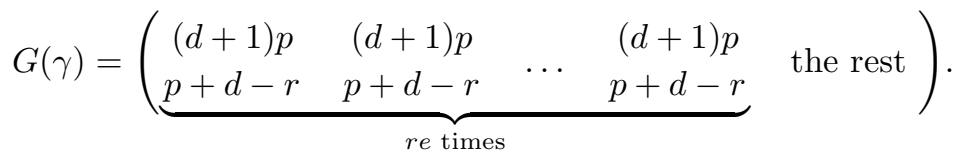

It follows from the Mullineux algorithm that $h(\gamma)=p+d-r$. Moreover, the $p$-edge of a partition has nodes in every row. In particular, the $p$-edge of a partition with $s$ rows has at least one node in the $s$ th row. It follows from the form of $G(\gamma)$ found above that the partitions $\gamma^{(1)}, \gamma^{(2)}, \ldots, \gamma^{(r e)}$ (defined at the end of Section 3) all have $s$ rows. Hence $\gamma_{s} \geq r e$.

Corollary 4.17. Let $m, e \in \mathbb{N}, \beta=\beta(m, p(p-1) e), \gamma=\mathbf{M}(\beta)$. Represent $m$ in the form

$$
m=(p-1) d+r, \quad d \in \mathbb{Z}, \quad 0<r \leq p-1 .
$$

Define $m_{1}=p+d-r$. Assume that $f \in \mathbb{Z}$ satisfies $0<f<$ re and set $\delta=\beta\left(m_{1}, f\right)$, $n=|\delta|$. Then

$$
D^{\delta} \in \operatorname{Irr}\left(D^{\gamma} \downarrow \Sigma_{n}\right)
$$


Proof. Follows immediately from Lemma 4.16, Proposition 4.14 (with $m=s=$ $p+d-r$ ), and Lemma 4.10 (with $\mu=\emptyset, m=p+d-r$ ).

Lemma 4.18. Let $e \in \mathbb{N}, \beta=\beta(p, p(p-1) e), \gamma=\mathbf{M}(\beta)$. Then

$$
\gamma=\left((p-1)+p e(p-1)^{2},(p e)^{p-1}\right) .
$$

Proof. One can easily see that the Mullineux symbol of $\beta$ is

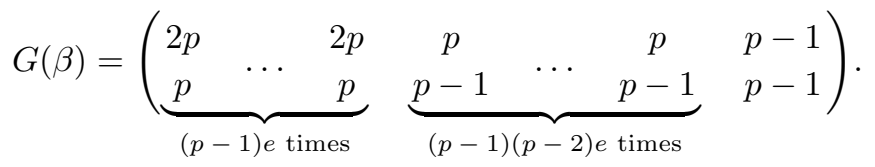

So, by Theorem 3.7, the Mullineux symbol of $\gamma$ is

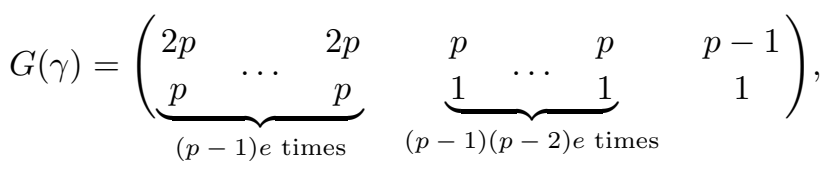

and the lemma follows.

Corollary 4.19. Let $p>2, n=(p-2)+p e(p-1)(p-2), e \in \mathbb{N}, \beta=\beta(p, p(p-1) e)$, $\gamma=\mathbf{M}(\beta)$. Then

$$
D^{(n)} \in \operatorname{Irr}\left(D^{\gamma} \downarrow \Sigma_{n}\right) .
$$

Proof. By Lemma 4.18, $\gamma=\left(\gamma_{1}, \gamma_{2}, \ldots, \gamma_{p}\right)$ where $\gamma_{1}=(p-1)+p e(p-1)^{2}, \gamma_{2}=$ $\gamma_{3}=\cdots=\gamma_{p}=p e$. Observe that $\gamma_{1}-\left(\gamma_{2}+\cdots+\gamma_{p}\right)=(p-1)+p e(p-1)(p-2)>2$. Moreover, $(n)=\beta\left(1, \gamma_{1}-\left(\gamma_{2}+\cdots+\gamma_{p}\right)-1\right)$. Now, by Proposition 4.14 (with $m=1$, $\lambda=\gamma)$ we have the desired result.

\section{INDUCTIVE SYSTEMS FOR THE FINITARY SYMMETRIC GROUP}

Now we apply the general notions of Section 2 to the case $G=\Sigma_{\infty}=\bigcup_{i=1}^{\infty} \Sigma_{i}$.

Definition 5.1. Let $\Phi=\left\{\Phi_{i}\right\}_{i \in \mathbb{N}}$ be an inductive system for $\Sigma_{\infty}$. We define its height $h(\Phi)$ as

$$
h(\Phi)=\sup \left\{h(\lambda) \mid D^{\lambda} \in \Phi_{i} \quad \text { for some } i \in \mathbb{N}\right\}
$$

(see (1)).

Proposition 5.2. Let $\Phi=\left\{\Phi_{i}\right\}_{i \in \mathbb{N}}$ be an inductive system for $\Sigma_{\infty}$. Assume that $h(\Phi)=+\infty$. Then $\Phi_{i}=\operatorname{Irr} \Sigma_{i} \quad$ for all $i \in \mathbb{N}$.

Proof. By assumption, for any $i \in \mathbb{N}$ there exist $j>i$ and $D^{\lambda} \in \Phi_{j}$ with $h(\lambda) \geq$ $i(p-1)$. Then

by Proposition 4.8 .

$$
\Phi_{i} \supseteq \operatorname{Irr}\left(D^{\lambda} \downarrow \Sigma_{i}\right)=\operatorname{Irr} \Sigma_{i},
$$

In [15] the second author has described all semisimple inductive systems for $\Sigma_{\infty}$ (see Definition 2.7). Surprisingly, it will turn out that the minimal inductive systems (i.e. those which correspond to the maximal ideals of $F \Sigma_{\infty}$ ) are exactly indecomposable semisimple inductive systems. We formulate some results from [15]. 
Recall the constant $\chi(\lambda)$ defined in (2). For $s=1,2, \ldots, p-1$ put

$$
\begin{aligned}
\Theta_{i}^{s} & =\left\{D^{\mu}|| \mu \mid=i, \quad h(\mu)=s, \quad \chi(\mu) \leq p\right\}, \\
\Omega_{i}^{s} & =\left\{D^{\mu}\left|\mu=\left(\mu_{1}, \mu_{2}, \ldots\right), \quad\right| \mu \mid=i, \quad h(\mu)<s, \quad \mu_{1} \leq p-s\right\} .
\end{aligned}
$$

Note that $\Theta_{i}^{s} \cap \Omega_{i}^{s}=\emptyset$ and $\Omega_{i}^{s}=\emptyset$ for $i>(p-s)(s-1)$. Set

$$
\Phi(s)_{i}=\Theta_{i}^{s} \sqcup \Omega_{i}^{s} .
$$

Theorem $5.3([15])$.

(i) $\Phi(s)=\left\{\Phi(s)_{i}\right\}_{i \in \mathbb{N}}, s=1,2, \ldots, p-1$, are all distinct semisimple minimal inductive systems for $\Sigma_{\infty}$.

(ii) Let $i \in \mathbb{N}$. If $j \gg i$, then for any $D^{\lambda} \in \Phi(s)_{j}$ we have

$$
\Phi(s)_{i}=\operatorname{Irr}\left(D^{\lambda} \downarrow \Sigma_{i}\right) .
$$

Proof. (ii) is proved in $[15,2.6,2.7]$, (i) is an immediate consequence of (ii) and $[15,2.8]$.

An important specific feature of the representation theory of symmetric groups is the presence of the sign module (denoted by sgn). Tensoring with sgn is often an effective trick.

Definition 5.4. Let $\Phi=\left\{\Phi_{i}\right\}_{i \in \mathbb{N}}$ be an inductive system for $\Sigma_{\infty}$. Define the inductive system $\Phi^{\sigma}=\left\{\Phi_{i}^{\sigma}\right\}_{i \in \mathbb{N}}$ by

$$
\Phi_{i}^{\sigma}=\left\{D^{\lambda} \otimes \operatorname{sgn} \mid D^{\lambda} \in \Phi_{i}\right\},
$$

where sgn is the sign representation of $\Sigma_{i}$.

$\Phi^{\sigma}$ is well defined in view of the following lemma.

Lemma 5.5. Let $\Phi=\left\{\Phi_{i}\right\}_{i \in \mathbb{N}}$ be an inductive system. Then

(i) $\Phi^{\sigma}=\left\{\Phi_{i}^{\sigma}\right\}_{i \in \mathbb{N}}$ is an inductive system;

(ii) $\Phi$ is minimal if and only if $\Phi^{\sigma}$ is so;

(iii) $\Phi$ is semisimple if and only if $\Phi^{\sigma}$ is so.

Proof. (i) and (iii) follow from the isomorphism

$$
(D \otimes \operatorname{sgn}) \downarrow \Sigma_{n-1} \cong\left(D \downarrow \Sigma_{n-1}\right) \otimes \operatorname{sgn}
$$

where $D$ is an $F \Sigma_{n}$-module and sgn in the left (resp., right) hand side means the sign representation of $\Sigma_{n}$ (resp., $\Sigma_{n-1}$ ). The second part follows from the equality $\left(\Phi^{\sigma}\right)^{\sigma}=\Phi$.

Lemma 5.6. Let $s \in\{1,2, \ldots, p-1\}$. Then $\Phi(s)^{\sigma}=\Phi(p-s)$.

Proof. By Lemma 5.5 (ii), (iii), $\Phi$ is minimal and semisimple if and only if $\Phi^{\sigma}$ is so. So Theorem 5.3 (i) implies $\Phi(s)^{\sigma}=\Phi(t)$ for some $t \in\{1,2, \ldots, p-1\}$. Note that $D^{\left((p e)^{s}\right)} \in \Phi(s)_{\text {pes }}$ for any $e \in \mathbb{N}$. However $h\left(\mathbf{M}\left((p e)^{s}\right)\right)=p-s$ by Lemma 4.16, which implies the demanded result.

Theorem 5.7. Let $p>2$, and $\Phi$ be an inductive system for $\Sigma_{\infty}$. Then $\Phi$ is minimal if and only if $\Phi=\Phi(s)$ for some $s \in\{1,2, \ldots, p-1\}$. 
Proof. The "if-part" follows from Theorem 5.3 (i).

Let $\Phi$ be minimal. If $h(\Phi)=+\infty$, then, by Proposition 5.2,

$$
\Phi_{i}=\operatorname{Irr} \Sigma_{i}, \quad i \in \mathbb{N} .
$$

However this system is not minimal (for example because it contains the trivial inductive system $\Phi(1))$. So $h(\Phi)=h<\infty$. It follows that for any $N \in \mathbb{N}$ there exist $i \in \mathbb{N}$ and $D^{\lambda} \in \Phi_{i}$ with $\lambda=\left(l_{1} \geq l_{2} \geq \cdots \geq l_{h} \geq 0\right)$ such that $l_{1}>N$. This shows that the set

$$
\begin{aligned}
V(\Phi)=\{v \in \mathbb{N} \mid & \text { for any } N \in \mathbb{N} \text { there exists } i \in \mathbb{N} \text { and } D^{\lambda} \in \Phi_{i} \\
& \text { with } \left.\lambda=\left(l_{1} \geq l_{2} \geq \cdots \geq l_{h} \geq 0\right) \text { such that } l_{v}>N\right\}
\end{aligned}
$$

is not empty. Since also $V(\Phi) \subseteq\{1,2, \ldots, h\}$ we can define

$$
m=m(\Phi)=\max V(\Phi) \text {. }
$$

It follows from the definition of $m$ that there exists $c \in \mathbb{N}$ such that

$$
\sum_{j=m+1}^{h} l_{j} \leq c
$$

for any $i \in \mathbb{N}$ and any $D^{\left(l_{1}, \ldots, l_{h}\right)} \in \Phi_{i}$. Let $N_{0} \geq c+2$. By definition of $m$, there exist $i \in \mathbb{N}$ and $D^{\left(l_{1}, \ldots, l_{h}\right)} \in \Phi_{i}$ such that $l_{m}>N_{0}$. Set

$$
\begin{aligned}
& f=l_{m}-\sum_{j=m+1}^{h} l_{j}-1, \\
& \beta=\beta(m, f), \quad n=|\beta| .
\end{aligned}
$$

By Proposition 4.14,

$$
D^{\beta} \in \operatorname{Irr}\left(D^{\left(l_{1}, \ldots, l_{h}\right)} \downarrow \Sigma_{n}\right) \subseteq \Phi_{n} .
$$

Note that $f>N_{0}-c-1$. Set

$$
\begin{aligned}
& f^{\prime}=N_{0}-c-1, \\
& \beta^{\prime}=\beta\left(m, f^{\prime}\right), \quad n^{\prime}=\left|\beta^{\prime}\right| .
\end{aligned}
$$

By Lemma 4.10,

$$
D^{\beta^{\prime}} \in \operatorname{Irr}\left(D^{\beta} \downarrow \Sigma_{n^{\prime}}\right) \subseteq \Phi_{n^{\prime}} .
$$

Choosing $N_{0}=c+2, c+3, \ldots$ (then $\left.f^{\prime}=1,2, \ldots\right)$ we conclude that

$$
D^{\beta(m, e)} \in \Phi_{|\beta(m, e)|} \text { for any } e \in \mathbb{N} .
$$

We call a number $t \in \mathbb{N}$ admissible for $\Phi$ if for any $N \in \mathbb{N}$ there is $f>N$ such that $D^{\beta(t, f)}$ belongs to $\Phi_{|\beta(t, f)|}$. By Lemma 4.10, we have

$$
\begin{aligned}
& t \in \mathbb{N} \text { is admissible for } \Phi \text { if and only if } \\
& D^{\beta(t, f)} \in \Phi_{|\beta(t, f)|} \text { for all } f \in \mathbb{N} .
\end{aligned}
$$

By (8), $m(\Phi)$ is admissible for $\Phi$. This allows us to define a constant $s(\Phi)$ as follows:

$$
s(\Phi)=\min \{t \in \mathbb{N} \mid t \text { is admissible for } \Phi\} .
$$

Let us consider the inductive system $\Phi^{\sigma}$ (see Definition 5.4). By Lemma 5.5 (ii), $\Phi^{\sigma}$ is minimal. So $s\left(\Phi^{\sigma}\right)$ is also well defined. We prove the following intermediate fact. 
$\left(^{*}\right)$ Assume that $s(\Phi) \leq s\left(\Phi^{\sigma}\right)$. Then $\Phi=\Phi(t)$ for some $t \in\{1,2, \ldots, p-1\}$.

Note that $\left(^{*}\right)$ immediately implies our theorem. Indeed, if $s(\Phi) \leq s\left(\Phi^{\sigma}\right)$, then $\left(^{*}\right)$ is just the theorem's claim. Let $s(\Phi)>s\left(\Phi^{\sigma}\right)$. Since $\left(\Phi^{\sigma}\right)^{\sigma}=\Phi$, (*) implies $^{*}$ $\Phi^{\sigma}=\Phi(t)$ for some $t \in\{1,2, \ldots, p-1\}$. Then $\Phi=\Phi(p-t)$ by Lemma 5.6.

Proof of $(*)$. Set $s=s(\Phi)$. We consider three cases.

(1) $s \leq p-1$. Then $\beta(s, f)=\left(f^{s}\right)$, and so $\chi(\beta(s, f))=s<p$. Hence $D^{\beta(s, f)} \in \Phi(s)_{|\beta(s, f)|}$ (see (6)). By (9), the modules $D^{\beta(s, f)}, f \in \mathbb{N}$, belong to the corresponding $\Phi_{|\beta(s, f)|}$. It now follows from Theorem 5.3 (ii) that $\Phi(s) \subseteq \Phi$. Since $\Phi$ is minimal, we have $\Phi=\Phi(s)$.

(2) $s=p$. Set $\beta(e)=\beta(p, p(p-1) e)$. By $(9)$,

$$
D^{\beta(e)} \in \Phi_{|\beta(e)|}
$$

for any $e \in \mathbb{N}$. Set $n(e)=(p-2)+e p(p-1)(p-2)$. Then, by Corollary 4.19,

$$
D^{(n(e))} \in \Phi_{n(e)}^{\sigma} .
$$

Note that $(n)=\beta(n, 1)$. So $n(e) \rightarrow+\infty$ as $e \rightarrow+\infty$ implies that 1 is admissible for $\Phi^{\sigma}$. This contradicts the assumption $s\left(\Phi^{\sigma}\right) \geq s(\Phi)$.

(3) $s>p$. Represent $s$ in the form

$$
s=(p-1) d+r, \quad d \in \mathbb{Z}, \quad 0<r \leq p-1 .
$$

By (9),

$$
D^{\beta(s, p(p-1) e)} \in \Phi_{|\beta(s, p(p-1) e)|} \quad \text { for any } e \in \mathbb{N} .
$$

By Corollary 4.17,

$$
D^{\beta(p+d-r, f)} \in \Phi_{|\beta(p+d-r, f)|}^{\sigma}
$$

for any $f<r e$. This shows that $p+d-r$ is admissible for $\Phi^{\sigma}$, whence $s\left(\Phi^{\sigma}\right) \leq$ $p+d-r$. However, this contradicts the assumption $s\left(\Phi^{\sigma}\right) \geq s(\Phi)$ because

$$
p+d-r<(p-1) d+r=s
$$

when $s>p$. The contradiction obtained completes the proof of $\left(^{*}\right)$ and the theorem.

Corollary 5.8. Let $p>2$. Set

$$
I(s)_{n}=\bigcap_{D \in \Phi(s)_{n}} \operatorname{Ann}_{F \Sigma_{n}}(D), \quad s=1, \ldots, p-1, \quad n \in \mathbb{N} .
$$

Then $I(s)_{1} \subset I(s)_{2} \subset \ldots$ Moreover,

$$
I(s)=\bigcup_{n=1}^{\infty} I(s)_{n}, \quad s=1, \ldots, p-1
$$

are exactly all distinct maximal ideals of $F \Sigma_{\infty}$, and $I(s) \cap F \Sigma_{n}=I(s)_{n}$.

Proof. In view of Proposition 2.6 (ii) and Theorem 5.7, the maximal ideals of $F \Sigma_{\infty}$ are exactly $I(\Phi(s)), s=1,2, \ldots, p-1$. However, the inductive systems $\Phi(s)$ are semisimple. So the result follows from 2.8. 


\section{INDUCTIVE SYSTEMS FOR THE FINITARY ALTERNATING GROUP}

Throughout the section we assume that $p>2$.

We first briefly discuss how to label irreducible $F A_{n}$-modules. It follows easily from the Clifford theory $[3,49.2]$ (see [4]) that the restriction $D^{\lambda} \downarrow_{A_{n}}^{\Sigma_{n}}$ is either irreducible or splits into a direct sum of two irreducibles. In the former case we write

and in the latter case we write

$$
D^{\lambda} \downarrow_{A_{n}}^{\Sigma_{n}}=E^{\lambda}
$$

$$
D^{\lambda} \downarrow_{A_{n}}^{\Sigma_{n}}=E_{+}^{\lambda} \oplus E_{-}^{\lambda} .
$$

It is of importance that $D^{\lambda} \downarrow_{A_{n}}^{\Sigma_{n}}$ is irreducible provided $\lambda \neq \mathbf{M}(\lambda)$. (In fact, if $F$ is a splitting field for $A_{n}$, then $D^{\lambda} \downarrow_{A_{n}}^{\Sigma_{n}}$ is irreducible if and only if $\lambda \neq \mathbf{M}(\lambda)$. However we want to work over an arbitrary field.) Every irreducible $F A_{n}$-module is of the form $E^{\lambda}$ or $E_{ \pm}^{\mu}$. Furthermore, $E_{+}^{\lambda} ¥ E_{-}^{\lambda}, E_{ \pm}^{\lambda} ¥ E_{ \pm}^{\mu}, E^{\lambda} ¥ E_{ \pm}^{\mu}$, and $E^{\lambda} \cong E^{\mu}$ if and only if $\lambda=\mathbf{M}(\mu)$ (for all permissible $\lambda \neq \mu$ ).

The following result was first proved in [17].

Lemma 6.1. [17] Let $\lambda \in \mathcal{P}_{n}$, and

$$
\operatorname{cont}(\lambda)=\left(c_{0}, c_{1}, \ldots, c_{p-1}\right) \text {. }
$$

Then

$$
\operatorname{cont}(\mathbf{M}(\lambda))=\left(d_{0}, d_{1}, \ldots, d_{p-1}\right)
$$

where $d_{0}=c_{0}$ and $d_{i}=c_{p-i}$ for $i=1,2, \ldots, p-1$.

Lemma 6.2. Let $p>2$. If $\lambda$ is a Young diagram with $\operatorname{cont}(\lambda)=\left(c_{0}, c_{1}, \ldots, c_{p-1}\right)$, then $c_{0} \leq c_{p-1}+1$.

Proof. This is obvious.

Lemma 6.3. Let $\lambda \in \mathcal{P}_{n}, \nu \in \mathcal{P}_{n+1}$ be such that

$$
D^{\lambda} \downarrow_{A_{n}}^{\Sigma_{n}}=E_{+}^{\lambda} \oplus E_{-}^{\lambda} \quad \text { and } \quad D^{\nu} \downarrow_{A_{n+1}}^{\Sigma_{n+1}}=E_{+}^{\nu} \oplus E_{-}^{\nu} .
$$

Assume that $E_{+}^{\lambda} \in \operatorname{Irr}\left(E_{+}^{\nu} \downarrow_{A_{n}}^{A_{n+1}}\right)$ or $E_{+}^{\lambda} \in \operatorname{Irr}\left(E_{-}^{\nu} \downarrow_{A_{n}}^{A_{n+1}}\right)$. Then $D^{\lambda} \in \operatorname{Irr}\left(D^{\nu} \downarrow_{\Sigma_{n}}^{\Sigma_{n+1}}\right)$.

Proof. If $D^{\lambda} \notin \operatorname{Irr}\left(D^{\nu} \downarrow_{\Sigma_{n}}^{\Sigma_{n+1}}\right)$, then

$E_{+}^{\lambda} \notin \operatorname{Irr}\left(\left(D^{\nu} \downarrow_{\Sigma_{n}}^{\Sigma_{n+1}}\right) \downarrow_{A_{n}}^{\Sigma_{n}}\right)=\operatorname{Irr}\left(\left(D^{\nu} \downarrow_{A_{n+1}}^{\Sigma_{n+1}}\right) \downarrow_{A_{n}}^{A_{n+1}}\right)=\operatorname{Irr}\left(E_{+}^{\nu} \downarrow_{A_{n}}^{A_{n+1}}\right) \cup \operatorname{Irr}\left(E_{-\downarrow}^{\nu} \downarrow_{A_{n}}^{A_{n+1}}\right)$,

giving a contradiction.

Lemma 6.4. Let $p>2$, and $\lambda \in \mathcal{P}_{n}$ be such that $D^{\lambda} \downarrow_{A_{n}}^{\Sigma_{n}}=E_{+}^{\lambda} \oplus E_{-}^{\lambda}$. Assume $\Phi=\left\{\Phi_{i}\right\}_{i \in \mathbb{N}}$ is an inductive system for $A_{\infty}$. Then $E_{+}^{\lambda} \in \Phi_{n}$ if and only if $E_{-}^{\lambda} \in \Phi_{n}$.

Proof. Assume $E_{+}^{\lambda} \in \Phi_{n}$ and show that $E_{-}^{\lambda} \in \Phi_{n}$ (the implication $E_{-}^{\lambda} \in \Phi_{n} \Rightarrow$ $E_{+}^{\lambda} \in \Phi_{n}$ is obtained similarly). Note that $\mathbf{M}(\lambda)=\lambda$. We shall prove the following intermediate fact.

(**) There is $k>n$ and $E^{\mu} \in \Phi_{k}$ such that $E_{+}^{\lambda} \in \operatorname{Irr}\left(E^{\mu} \downarrow_{A_{n}}^{A_{k}}\right)$.

This will imply the lemma. Indeed, $E^{\mu}=D^{\mu} \downarrow_{A_{k}}^{\Sigma_{k}}$. Therefore

$$
E^{\mu} \downarrow_{A_{n}}^{A_{k}} \cong\left(D^{\mu} \downarrow_{\Sigma_{n}}^{\Sigma_{k}}\right) \downarrow_{A_{n}}^{\Sigma_{n}}
$$

So $D^{\lambda} \in \operatorname{Irr}\left(D^{\mu} \downarrow_{\Sigma_{n}}^{\Sigma_{k}}\right)$ because otherwise we would have $E_{+}^{\lambda} \notin \operatorname{Irr}\left(E^{\mu} \downarrow_{A_{n}}^{A_{k}}\right)$. It now follows from (10) that $E_{-}^{\lambda} \in \operatorname{Irr}\left(E^{\mu} \downarrow_{A_{n}}^{A_{k}}\right)$. 
We now prove $(* *)$. Let $\operatorname{cont}(\lambda)=\left(c_{0}, c_{1}, \ldots, c_{p-1}\right)$. Since $\Phi$ is an inductive system there exists an irreducible module $E \in \Phi_{n+1}$ such that $E_{+}^{\lambda} \in \operatorname{Irr}\left(E \downarrow_{A_{n}}^{A_{n+1}}\right)$. If $E=E^{\nu}$, we can take $\mu=\nu, k=n+1$. Otherwise $E=E_{+}^{\nu}$ or $E_{-}^{\nu}$ for some $\nu$ with $\mathbf{M}(\nu)=\nu$. In view of Lemma 6.3, we have

$$
D^{\lambda} \in \operatorname{Irr}\left(D^{\nu} \downarrow_{\Sigma_{n}}^{\Sigma_{n+1}}\right) \text {. }
$$

So by Lemma 4.15,

$$
\operatorname{cont}(\nu)=\left(c_{0}, \ldots, c_{j}+1, \ldots, c_{p-1}\right)
$$

for some $j \in\{0,1, \ldots, p-1\}$. Since $\mathbf{M}(\lambda)=\lambda$ and $\mathbf{M}(\nu)=\nu$, Lemma 6.1 forces $j=0$. Thus,

$$
\operatorname{cont}(\nu)=\left(c_{0}+1, c_{1}, \ldots, c_{p-1}\right) .
$$

Repeating this argument $i$ times we either arrive to a module $E^{\mu} \in \Phi_{n+i}$ such that $E_{+}^{\lambda} \in \operatorname{Irr}\left(E^{\mu} \downarrow_{A_{n}}^{A_{n+i}}\right)$ or we get a partition $\nu$ with

$$
\operatorname{cont}(\nu)=\left(c_{0}+i, c_{1}, \ldots, c_{p-1}\right) .
$$

However, Lemma 6.2 shows that $i$ cannot be too large.

Lemma 6.5. Let $\Phi=\left\{\Phi_{i}\right\}_{i \in \mathbb{N}}$ be an inductive system for $\Sigma_{\infty}$. Set

$$
\bar{\Phi}_{i}=\operatorname{Irr}\left(\Phi_{i} \downarrow_{A_{i}}^{\Sigma_{i}}\right) .
$$

Then $\bar{\Phi}=\left\{\bar{\Phi}_{i}\right\}_{i \in \mathbb{N}}$ is an inductive system for $A_{\infty}$.

Proof. Note that

$$
\begin{aligned}
& \operatorname{Irr}\left(\bar{\Phi}_{i} \downarrow_{A_{i-1}}^{A_{i}}\right)=\operatorname{Irr}\left(\operatorname{Irr}\left(\Phi_{i} \downarrow_{A_{i}}^{\Sigma_{i}}\right) \downarrow_{A_{i-1}}^{A_{i}}\right)=\operatorname{Irr}\left(\Phi_{i} \downarrow_{A_{i-1}}^{\Sigma_{i}}\right) \\
& =\operatorname{Irr}\left(\operatorname{Irr}\left(\Phi_{i} \downarrow_{\Sigma_{i-1}}^{\Sigma_{i}}\right) \downarrow_{A_{i-1}}^{\Sigma_{i-1}}\right)=\operatorname{Irr}\left(\Phi_{i-1} \downarrow_{A_{i-1}}^{\Sigma_{i-1}}\right)=\bar{\Phi}_{i-1},
\end{aligned}
$$

as demanded.

Lemma 6.5 allows us to give the following definition.

Definition 6.6. Let $\Phi$ be an inductive system for $\Sigma_{\infty}$. Define the inductive system $\Phi \downarrow A_{\infty}$ for $A_{\infty}$ as $\Phi \downarrow A_{\infty}=\bar{\Phi}$ (see Lemma 6.5).

Definition 6.7. Let $S \subseteq \operatorname{Irr}\left(\Sigma_{n}\right)$. Define the set $S^{\sigma} \subseteq \operatorname{Irr}\left(\Sigma_{n}\right)$ as

$$
S^{\sigma}=\{D \otimes \operatorname{sgn} \mid D \in S\} .
$$

Lemma 6.8. Let $S, T \subseteq \operatorname{Irr}\left(\Sigma_{n}\right), S^{\sigma}=S, T^{\sigma}=T$. If $\operatorname{Irr}\left(S \downarrow_{A_{n}}^{\Sigma_{n}}\right)=\operatorname{Irr}\left(T \downarrow_{A_{n}}^{\Sigma_{n}}\right)$, then $S=T$.

Proof. Assume $D^{\lambda} \in S$ but $D^{\lambda} \notin T$. Then $D^{\mathbf{M}(\lambda)} \notin T$. Hence $E \in \operatorname{Irr}\left(S \downarrow_{A_{n}}^{\Sigma_{n}}\right)$ but $E \notin \operatorname{Irr}\left(T \downarrow_{A_{n}}^{\Sigma_{n}}\right)$ where $E=E^{\lambda}$ or $E=E_{+}^{\lambda}$. The contradiction obtained shows that $S \subseteq T$. Similarly, $T \subseteq S$.

Lemma 6.9. Let $S \subseteq \operatorname{Irr}\left(\Sigma_{n}\right)$. If $S^{\sigma}=S$, then $\left(\operatorname{Irr}\left(S \downarrow \Sigma_{n-1}\right)\right)^{\sigma}=\operatorname{Irr}\left(S \downarrow \Sigma_{n-1}\right)$.

Proof. Follows from (7).

Theorem 6.10. Let $p>2$. The map $\Phi \rightarrow \Phi \downarrow A_{\infty}$ defines an isomorphism between the poset of the inductive systems $\Phi$ for $\Sigma_{\infty}$ such that $\Phi^{\sigma}=\Phi$ and the poset of the inductive systems for $A_{\infty}$. 
Proof. The map is injective by Lemma 6.8. We construct a right inverse map. Let $\Psi=\left\{\Psi_{i}\right\}_{i \in \mathbb{N}}$ be an inductive system for $A_{\infty}$. Set

$$
\hat{\Psi}_{i}=\left\{D^{\lambda} \in \operatorname{Irr} \Sigma_{i} \mid \operatorname{Irr}\left(D^{\lambda} \downarrow_{A_{i}}^{\Sigma_{i}}\right) \subseteq \Psi_{i}\right\}, \quad i \in \mathbb{N} .
$$

We first show that

$$
\Psi_{i}=\operatorname{Irr}\left(\hat{\Psi}_{i} \downarrow_{A_{i}}^{\Sigma_{i}}\right), \quad i \in \mathbb{N} .
$$

Indeed, $\Psi_{i} \supseteq \operatorname{Irr}\left(\hat{\Psi}_{i} \downarrow_{A_{i}}^{\Sigma_{i}}\right)$ by definition of $\hat{\Psi}_{i}$. On the other hand, let $E \in \Psi_{i}$. There are two cases:

(1) $E=E^{\lambda}$;

(2) $E=E_{+}^{\lambda}$ or $E=E_{-}^{\lambda}$.

In the case (1), $E=D^{\lambda} \downarrow_{A_{i}}^{\Sigma_{i}}$; hence $D^{\lambda} \in \hat{\Psi}_{i}$ and $E \in \operatorname{Irr}\left(\hat{\Psi}_{i} \downarrow_{A_{i}}^{\Sigma_{i}}\right)$. In the case (2), by Lemma 6.4 , both $E_{+}^{\lambda}$ and $E_{-}^{\lambda}$ belong to $\Psi$. So $D^{\lambda} \downarrow_{A_{i}}^{\Sigma_{i}}=E_{+}^{\lambda} \oplus E_{-}^{\lambda}$ implies that $D^{\lambda} \in \hat{\Psi}_{i}$; hence $E \in \operatorname{Irr}\left(\hat{\Psi}_{i} \downarrow_{A_{i}}^{\Sigma_{i}}\right)$.

It follows from the definition of $\hat{\Psi}_{j}$ that

$$
\left(\hat{\Psi}_{j}\right)^{\sigma}=\hat{\Psi}_{j}, \quad j \in \mathbb{N} .
$$

Taking $j=i-1$ gives

$$
\left(\hat{\Psi}_{i-1}\right)^{\sigma}=\hat{\Psi}_{i-1} .
$$

Taking $j=i$ and using Lemma 6.9, we get

$$
\left(\operatorname{Irr}\left(\hat{\Psi}_{i} \downarrow_{\Sigma_{i-1}}^{\Sigma_{i}}\right)\right)^{\sigma}=\operatorname{Irr}\left(\hat{\Psi}_{i} \downarrow_{\Sigma_{i-1}}^{\Sigma_{i}}\right) .
$$

Now we show that

$$
\operatorname{Irr}\left(\operatorname{Irr}\left(\hat{\Psi}_{i} \downarrow_{\Sigma_{i-1}}^{\Sigma_{i}}\right) \downarrow_{A_{i-1}}^{\Sigma_{i-1}}\right)=\operatorname{Irr}\left(\hat{\Psi}_{i-1} \downarrow_{A_{i-1}}^{\Sigma_{i-1}}\right) .
$$

Indeed, using (11) twice, we obtain

$$
\begin{gathered}
\operatorname{Irr}\left(\operatorname{Irr}\left(\hat{\Psi}_{i} \downarrow_{\Sigma_{i-1}}^{\Sigma_{i}}\right) \downarrow_{A_{i-1}}^{\Sigma_{i-1}}\right)=\operatorname{Irr}\left(\hat{\Psi}_{i} \downarrow_{A_{i-1}}^{\Sigma_{i}}\right)=\operatorname{Irr}\left(\operatorname{Irr}\left(\hat{\Psi}_{i} \downarrow_{A_{i}}^{\Sigma_{i}}\right) \downarrow_{A_{i-1}}^{A_{i}}\right) \\
=\operatorname{Irr}\left(\Psi_{i \downarrow_{A_{i-1}}}^{A_{i}}\right)=\Psi_{i-1}=\operatorname{Irr}\left(\hat{\Psi}_{i-1} \downarrow_{A_{i-1}}^{\Sigma_{i-1}}\right) .
\end{gathered}
$$

In view of Lemma 6.8, equations (13), (14) and (15) imply

$$
\operatorname{Irr}\left(\hat{\Psi}_{i} \downarrow_{\Sigma_{i-1}}^{\Sigma_{i}}\right)=\hat{\Psi}_{i-1},
$$

i.e. $\hat{\Psi}$ is an inductive system. Moreover, (11) shows that $\hat{\Psi} \downarrow A_{\infty}=\Psi$. It remains to note that $\Psi \subseteq \Phi$ if and only if $\hat{\Psi} \subseteq \hat{\Phi}$.

Theorem 6.11. Let $p>2$. The inductive systems

$$
\Phi(s) \downarrow A_{\infty}, \quad s=1,2, \ldots, \frac{p-1}{2},
$$

are exactly all distinct minimal inductive systems for $A_{\infty}$.

Proof. Let $\mathcal{S}$ be the set of all inductive systems $\Phi$ for $\Sigma_{\infty}$ which satisfy the following two conditions:

(1) $\Phi^{\sigma}=\Phi$.

(2) If $\Psi$ is an inductive system for $\Sigma_{\infty}, \Psi \subseteq \Phi$, and $\Psi^{\sigma}=\Psi$, then $\Psi=\Phi$.

By Theorem 6.10,

$$
\left\{\Phi \downarrow A_{\infty} \mid \Phi \in \mathcal{S}\right\}
$$

is the set of all distinct minimal inductive systems for $A_{\infty}$. 
We claim that $\Phi \in \mathcal{S}$ if and only if $\Phi=\Phi(s) \cup \Phi(s)^{\sigma}$ for some $s \in\{1,2, \ldots, p-1\}$ (see (6)). Indeed, let $\Phi \in \mathcal{S}$. By Theorem 5.7, $\{\Phi(s) \mid 1 \leq s \leq p-1\}$ are exactly all minimal inductive systems for $\Sigma_{\infty}$. So $\Phi$ contains some $\Phi(s)$. Since $\Phi^{\sigma}=\Phi$, we also have $\Phi \supseteq \Phi(s)^{\sigma}$. Thus $\Phi(s) \cup \Phi(s)^{\sigma} \subseteq \Phi$. Since $\left(\Phi(s) \cup \Phi(s)^{\sigma}\right)^{\sigma}=\Phi(s) \cup \Phi(s)^{\sigma}$ and $\Phi \in \mathcal{S}$, we conclude that $\Phi=\Phi(s) \cup \Phi(s)^{\sigma}$.

On the other hand, if $\Phi(s) \cup \Phi(s)^{\sigma} \notin \mathcal{S}$, then there exists an inductive system $\Phi$ with $\Phi^{\sigma}=\Phi$ such that $\Phi \underset{\neq}{\subset} \Phi(s) \cup \Phi(s)^{\sigma}$. As above, we find $t \in\{1,2, \ldots, p-1\}$ such that $\Phi(t) \cup \Phi(t)^{\sigma} \subseteq \Phi$. Therefore,

$$
\Phi(t) \cup \Phi(t)^{\sigma} \underset{\neq}{\subset} \Phi(s) \cup \Phi(s)^{\sigma}
$$

for some $s, t \in\{1,2, \ldots, p-1\}$, which is false in view of Lemma 5.6 and the definition of $\Phi(u)$. Since $\left(\Phi(s) \cup \Phi(s)^{\sigma}\right) \downarrow A_{\infty}=\Phi(s) \downarrow A_{\infty}$ it remains to use Lemma 5.6 another time.

Lemma 6.12. The inductive systems $\Phi(s) \downarrow A_{\infty}, s=1,2, \ldots, \frac{p-1}{2}$, are semisimple. Proof. This follows immediately from the fact that $\Phi(s)$ are semisimple.

Corollary 6.13. Let $p>2$. Set

$$
J(t)_{n}=\bigcap_{D \in \Phi(t)_{n}} \operatorname{Ann}_{F A_{n}}\left(D \downarrow A_{n}\right), \quad t=1, \ldots, \frac{p-1}{2}, \quad n \in \mathbb{N} .
$$

Then

(i) $J(t)_{1} \subset J(t)_{2} \subset \ldots$ Moreover,

$$
J(t)=\bigcup_{n=1}^{\infty} J(t)_{n}, \quad t=1, \ldots, \frac{p-1}{2},
$$

are exactly all distinct maximal ideals of $F A_{\infty}$, and $J(t) \cap F A_{n}=J(t)_{n}$.

(ii) $I(s) \cap F A_{\infty}=J(t)$ where $t=\min (s, p-s), s=1, \ldots, p-1$.

Proof. The proof of (i) is similar to that of Corollary 5.8.

(ii) Let $t=\min (s, p-s)$. In view of Corollary 5.8, Lemma 5.6 and (i), we have

$$
\begin{aligned}
& I(s) \cap F A_{i}=\left(I(s) \cap F \Sigma_{i}\right) \cap F A_{i}=\left(\bigcap_{D \in \Phi(s)_{i}} \operatorname{Ann}_{F \Sigma_{i}}(D)\right) \cap F A_{i} \\
& =\bigcap_{D \in \Phi(s)_{i}} \operatorname{Ann}_{F A_{i}}\left(D \downarrow A_{i}\right)=\bigcap_{D \in \Phi(t)_{i}} \operatorname{Ann}_{F A_{i}}\left(D \downarrow A_{i}\right)=J(t)_{i}=J(t) \cap F A_{i}
\end{aligned}
$$

for any $i \in \mathbb{N}$. This implies (ii).

Remark. In view of Corollaries 5.8 and 6.13 (i),

$$
I(s)=\bigcup_{j>(p-s)(s-1)} I(s)_{j}
$$

and

$$
J(t)=\bigcup_{i>(p-t)(t-1)} J(t)_{i}
$$

This allows one to describe the ideals $I(s)$ and $J(t)$ in a more compact form, because $\Omega_{i}^{s}=\emptyset$ for $i>(p-s)(s-1)$, and so

$$
\Psi(s)_{i}=\Theta_{i}^{s},
$$

see (6). This is done in Theorem 1.1. 


\section{REFERENCES}

1. S. A. Amitsur, The polynomial identities of associative rings, in "Noetherian Rings and Rings with Polynomial Identities", Proc. Conf. Univ. Durham, 1979, pp. 1-38.

2. C. Bessenrodt and J.B. Olsson. On residue symbols and the Mullineux conjecture, J. Algebraic Combin. 7 (1998), 227-251. CMP 98:10

3. C. W. Curtis and I. Reiner, "Representation Theory of Finite Groups and Associative Algebras". Interscience (Wiley), New York / London, 1962. MR 26:2519

4. B. Ford, Irreducible representations of the alternating groups in odd characteristic, Proc. Amer. Math. Soc. 125 (1997), 267-308. MR 97d:20008

5. B. Ford and A. Kleshchev, A proof of the Mullineux conjecture, Math. Z. 226 (1997), 257-308. CMP 98:03

6. E. Formanek and D. Lawrence, The group algebra of the infinite symmetric group, Israel J. Math. 23 (1976), 325-331. MR 54:5324

7. E. Formanek and C. Procesi, Mumford's conjecture for the general linear group, Adv. in Math. 19 (1976), 292-305. MR 53:8081

8. G. D. James, On the decomposition matrices of the symmetric groups, II, J. Algebra 43 (1976), 45-54. MR 55:3057b

9. G. D. James, On a conjecture of Carter concerning irreducible Specht modules, Math. Proc. Camb. Phil. Soc. 83 (1978), 11-17. MR 57:3234

10. G. D. James, "The Representation Theory of the Symmetric Groups", Lecture Notes in Mathematics, Vol. 682, Springer-Verlag, Berlin / New York, 1978. MR 80g:20019

11. G. D. James and A. Kerber, "The Representation Theory of the Symmetric Group", Encyclopedia of Math. and its Applications, Vol. 16, Addison-Wesley, 1981. MR 83k:20003

12. G. D. James and G. E. Murphy, The determinant of the Gram matrix for a Specht module, J. Algebra 59 (1979), 222-235. MR 82j:20025

13. A. S. Kleshchev, Branching rules for modular representations of symmetric groups, II, J. Reine Angew. Math. 459 (1995), 163-212. MR 96m:20019b

14. A. S. Kleshchev, Branching rules for modular representations of symmetric groups, III, J. London Math. Soc. (2) 54(1996), 25-38. MR 96m:20019c

15. A. S. Kleshchev, Completely splittable representations of symmetric groups, J. Algebra 181 (1996), 584-592. MR 97a:20017

16. A. S. Kleshchev, On decomposition numbers and branching coefficients for symmetric and special linear groups, Proc. London Math. Soc. (3) 75 (1997), 497-558. CMP 97:17

17. G. Mullineux, On the $p$-cores of $p$-regular diagrams, J. London Math. Soc. (2), 20 (1979), 222-225. MR 81a:20017

18. Yu. P. Razmyslov, Trace identities of full matrix algebras over a field of characteristic zero, Math. USSR. Izvestiya 8 (1974), 727-760. MR 58:22158

19. Yu. P. Razmyslov, "Identities of Algebras and Their Representations", Nauka, Moscow, 1989; English transl., Amer. Math. Soc., Providence, RI, 1994. MR 91c:17022; MR 95i:16022

20. L. H. Rowen, "Polynomial Identities in Ring Theory", Academic Press, New York, 1980. MR 82a: 16021

21. S. K. Sehgal and A. E. Zalesskii, Induced modules and some arithmetic invariants of the finitary symmetric groups, Nova J. Algebra Geom. 2 (1993), 89-105. MR 94m:20010

22. A. E. Zalesskii, Modular group rings of the finitary symmetric groups, Israel J. Math. 96 (1996), part B, 609-621. MR 97k:20012

23. A. E. Zalesskii, Group rings of simple locally finite groups, in "Finite and locally finite groups", Kluwer, Dordrecht, 1995, 219-246. MR 96k:16044

Institute of Mathematics, Academy of Sciences of Belarus, Surganova 11, Minsk, 220072, BeLARUs

E-mail address: baranov@im.bas-net.by

Department of Mathematics, University of Oregon, Eugene, Oregon 97403

E-mail address: klesh@math.uoregon.edu 